\title{
COMMUTATION OF VARIATION AND DUAL PROJECTION
}

\author{
DAVID NEAL
}

(Communicated by Richard Durrett)

\begin{abstract}
For a raw process of integrable variation $V$, taking values in a Banach space $E$ having the Radon-Nikodyn property, the variation of the predictable (optional) dual projection is the predictable (optional) dual projection of the variation. An analogous result holds for the associated stochastic measures. The result is applied to the stochastic integral of a real, optional process $H$ with respect to $V$ when $V$ is adapted.
\end{abstract}

\section{INTRODUCTION}

In a series of articles [4-7], Dinculeanu has detailed the theory of Banachvalued stochastic processes. In particular, given a raw process of integrable variation $V$, the existence of predictable and optional dual projections, denoted by $V^{p}$ and $V^{o}$ respectively, is established [6, Theorems 14 and 15]. The variations of these processes satisfy the inequalities $\left|V^{p}\right| \leq|V|^{p}$ and $\left|V^{o}\right| \leq$ $|V|^{\circ}[6]$.

In this article, we use an alternate construction of the dual projection to show that in fact $\left|V^{p}\right|=|V|^{p}$ and $\left|V^{o}\right|=|V|^{o}$. We obtain analogous equalities for the associated stochastic measures $\mu_{p}$ and $\mu_{o}$, which improves upon the inequalities in Lemma 1 from [7]. Lastly, we apply this commutation result to provide a sufficient condition for a real optional process $H$ to be stochastically integrable with respect to an adapted process of integrable variation $V$.

Throughout, we let $(\Omega, \mathscr{F}, P)$ be a probability space with a filtration $(\mathscr{F})$ which satisfies the usual conditions. We let $E$ be a Banach space which has the Radon-Nikodym property and let $V$ be an $E$-valued, raw process of integrable variation which is also separably valued.

\section{THE VARIATION OF THE PROJECTION}

We proceed immediately with the result on commutation of variation and dual projection.

Received by the editors August 11,1993; the contents of this paper were presented at the January 1994 meeting of the AMS in Cincinnati.

1991 Mathematics Subject Classification. Primary 60G07; Secondary 60H05.

Key words and phrases. Dual projections, variation, stochastic measures, optional stochastic integral. 
Theorem 1. The variation of the predictable (resp. optional) dual projection of $V$ equals the predictable (resp. optional) dual projection of the variation. That is, $\left|V^{p}\right|=|V|^{p} \quad\left(\right.$ resp. $\left.\left|V^{o}\right|=|V|^{o}\right)$.

Proof. We prove the result for the predictable projection. The other case is analogous. We define a stochastic measure $\mu$ on the predictable $\sigma$-algebra by $\mu(\cdot)=$ $E\left[\int^{\infty} 1_{(\cdot)} d V_{s}\right]$ and note that its variation is given by $|\mu|(\cdot)=E\left[\int^{\infty} 1_{(\cdot)} d|V|_{s}\right]$ [4, Theorem 3.2]. Since $\mu$ is absolutely continuous with respect to $|\mu|$, we let $Q=d \mu / d|\mu|$ be an $E$-valued predictable density such that $\|Q\|=1,|\mu|$ almost everywhere. Then for all predictable sets $B$,

$$
E\left[\int^{\infty} 1_{B} d V_{s}\right]=E\left[\int^{\infty} 1_{B} Q_{s} d|V|_{s}\right] .
$$

We now let $W=|V|^{p}$. Then for every real, bounded, measurable process $H$, with predictable projection ${ }^{p} H$,

$$
\begin{aligned}
E\left[\int^{\infty}{ }^{p} H_{s} d V_{s}\right] & =E\left[\int^{\infty}{ }^{p} H_{s} Q_{s} d|V|_{s}\right] \\
& =E\left[\int^{\infty}{ }^{p}\left(H_{s} Q_{s}\right) d|V|_{s}\right] \\
& =E\left[\int^{\infty} H_{s} Q_{s} d W_{s}\right] .
\end{aligned}
$$

Thus, $V_{t}^{p}=\int^{t} Q_{s} d W_{s}$. However, since $W$ is an increasing process, $d W_{s}$ defines a scalar-valued measure on $B\left(R^{+}\right)$(for each $w$ ). Hence, the total variation of $V^{p}$ is given by $\left|V^{p}\right|_{t}=\int^{t}\left\|Q_{s}\right\| d W_{s}[3, \S 10.9$, Theorem 6, p. 186].

On the other hand, since $\left|\left\|Q_{s}\right\|-1\right|$ is a predictable process,

$$
\begin{aligned}
E\left[\int^{\infty}\left|\left\|Q_{s}\right\|-1\right| d W_{s}\right] & =E\left[\int^{\infty}\left|\left\|Q_{s}\right\|-1\right| d|V|_{s}\right] \\
& =\int\left|\left\|Q_{s}\right\|-1\right| d|\mu|=0 ;
\end{aligned}
$$

hence, $\int^{\infty}\left|\left\|Q_{s}\right\|-1\right| d W_{s}=0$ a.e. It follows that $\int^{t}\left\|Q_{s}\right\| d W_{s}$ and $\int^{t} 1 d W_{s}$ are indistinguishable. Hence, $\left|V^{p}\right|=\int^{t} d W_{s}=|V|^{p}$.

Corollary. Let $E$ be separable and let $\mu$ be an $E$-valued $P$-measure on $B\left(R^{+}\right) \times$ $\mathscr{F}$ with finite variation $|\mu|$, with predictable (resp. optional) projection denoted by $\mu_{p}\left(\right.$ resp. $\left.\mu_{o}\right)$. Then, $\left|\mu_{p}\right|=|\mu|_{p}\left(\right.$ resp. $\left.\left|\mu_{o}\right|=|\mu|_{o}\right)$.

Proof. We let $V$ be the associated raw process of integrable variation associated with $\mu$ which satisfies the following [4, Theorems 4.1, 5.1]:

$$
\mu(\cdot)=E\left[\int^{\infty} 1_{(\cdot)} d V_{s}\right], \quad|\mu|(\cdot)=E\left[\int^{\infty} 1_{(\cdot)} d|V|_{s}\right] .
$$

Then since $V^{p}$ is the process associated with $\mu_{p}$, we have

$$
\begin{aligned}
\left|\mu_{p}\right|(\cdot) & =E\left[\int^{\infty} 1_{(\cdot)} d\left|V^{p}\right|_{s}\right] \\
& =E\left[\int^{\infty} 1_{(\cdot)} d|V|_{s}^{p}\right]=|\mu|_{p}(\cdot) .
\end{aligned}
$$

The argument is analogous for the optional projection. 


\section{OPTIONAL STOCHASTIC INTEGRATION}

As an application of Theorem 1, we shall prove a sufficient condition for a real-valued optional process $H$ to be stochastically integrable, as defined in [8] with respect to an adapted process of integrable variation $V$. We also describe the form of the stochastic integral $H \cdot V$.

Theorem 2. Let $H$ be a real-valued optional process such that ${ }^{p} H$ exists and let $V$ be an $E$-valued, adapted process of integrable variation. If $E\left[\int^{\infty}\left|H_{s}\right| d|V|_{s}\right]<$ $\infty$ and $E\left[\int^{\infty} p\left|H_{s}\right| d|V|_{s}\right]<\infty$, then $H \in \mathscr{L}_{1}\left(I_{V}\right)$ and the optional stochastic integral of $H$ with respect to $V$ is given by

$$
(H \cdot V)_{t}=\int^{t} H_{s} d V_{s}-\left(\int H_{s} d V_{s}\right)_{t}^{p}+\int^{t}{ }^{p} H_{s} d V_{s}^{p}
$$

Proof. From [8], the measure $I_{V}$ is defined on the optional $\sigma$-algebra $\mathscr{O}$ by $I_{V}(B)=\int^{\infty} 1_{B} d V_{s}-\left(\int^{\bullet} 1_{B} d V_{s}\right)_{\infty}^{p}+\int^{\infty} p\left(1_{B}\right) d V_{s}^{p}$, where $B \in \mathscr{O}$, and hence

$$
\left(1_{B} \cdot V\right)_{t}=\int 1_{B} 1_{[0, t]} d I_{V}=\int^{t} 1_{B} d V_{s}-\left(\int^{\cdot} 1_{B} d V_{s}\right)_{t}^{p}+\int^{t} p\left(1_{B}\right) d V_{s}^{p} .
$$

By additivity, the result holds for the generating class of processes $H$ of the form $1_{\left[S_{1}, T_{1}[\right.}+\cdots+1_{\left[S_{n}, T_{n}[\right.}$, where $S_{i}, T_{i}$ are stopping times with $S_{1} \leq T_{1} \leq$ $\cdots \leq S_{n} \leq T_{n}$. We shall use a monotone class argument to first show the result for bounded $H$, since if $H$ is bounded then ${ }^{p} H$ exists and is also bounded [2, VI.43]. Consequently, both $E\left[\int^{\infty}\left|H_{s}\right| d|V|_{s}\right]$ and $E\left[\int^{\infty} p\left|H_{s}\right| d|V|_{s}\right]$ are finite since $V$ is of integrable variation.

If the theorem holds for a nonnegative sequence $\left\{H^{n}\right\}$ which is uniformly bounded and increases to $H$, or for a sequence converging uniformly to $H$, then by dominated convergence $\left\{H^{n}\right\}$ will also converge to $H$ in the Lebesgue space $L_{1}\left(I_{V}\right)$. Thus, the $L_{1}(E)$-valued stochastic integrals $\left\{\left(H^{n} \cdot V\right)_{t}\right\}$ will converge for each $t$ to $(H \cdot V)_{t}$ since

$$
\begin{aligned}
\left\|\left(H^{n} \cdot V\right)_{t}-(H \cdot V)_{t}\right\|_{L_{1}(E)} & =\left\|\int\left(H^{n}-H\right) 1_{[0, t]} d I_{V}\right\|_{L_{1}(E)} \\
& \leq\left\|\left(H^{n}-H\right)\right\|_{L_{1}\left(I_{V}\right)} .
\end{aligned}
$$

We see that all three pieces of $H^{n} \cdot V$ will converge in $L_{1}(E)$ to the appropriate pieces of $H \cdot V$. For if we let $W_{t}^{n}=\int^{t}\left(H_{s}^{n}-H_{s}\right) d V_{s}$, then $E\left[\left\|W_{t}^{n}\right\|\right] \leq$ $E\left[\int^{\infty}\left|H_{s}^{n}-H_{s}\right| d|V|_{s}\right] \rightarrow 0$, by dominated convergence. Also,

$$
\begin{aligned}
E\left[\left\|\left(W^{n}\right)_{t}^{p}\right\|\right] & \leq E\left[\int^{\infty} d\left|\left(W^{n}\right)^{p}\right|_{s}\right] \\
& =E\left[\int^{\infty} d\left|W^{n}\right|_{s}^{p}\right] \\
& =E\left[\int^{\infty} d\left|W^{n}\right|_{s}\right] \\
& \leq E\left[\int^{\infty}\left|H_{s}^{n}-H_{s}\right| d|V|_{s}\right]
\end{aligned}
$$


hence, $\left(W^{n}\right)_{t}^{p} \rightarrow 0$ in $L_{1}(E)$. Moreover, since $V^{p}$ is also of integrable variation,

$$
\begin{aligned}
E\left[\left\|\int^{t}{ }^{p}\left(H^{n}-H\right)_{s} d V_{s}^{p}\right\|\right] & \leq E\left[\int^{\infty}\left|{ }^{p}\left(H^{n}-H\right)_{s}\right| d\left|V^{p}\right|_{s}\right] \\
& \leq E\left[\int^{\infty}{ }^{p}\left|H_{s}^{n}-H_{s}\right| d\left|V^{p}\right|_{s}\right] \\
& =E\left[\int^{\infty}\left|H_{s}^{n}-H_{s}\right| d\left|V^{p}\right|_{s}\right] \\
& \rightarrow 0 .
\end{aligned}
$$

Hence, in $L_{1}(E)$,

$$
\begin{aligned}
(H \cdot V)_{t} & =\lim _{n \rightarrow \infty}\left(H^{n} \cdot V\right)_{t}=\lim _{n \rightarrow \infty}\left[\int H_{s}^{t} d V_{s}-\left(\int H_{s}^{n} d V_{s}\right)_{t}^{p}+\int{ }^{t} H_{s}^{n} d V_{s}^{p}\right] \\
& =\int^{t} H_{s} d V_{s}-\left(\int H_{s} d V_{s}\right)_{t}^{p}+\int^{t}{ }^{t} H_{s} d V_{s}^{p} .
\end{aligned}
$$

We thus obtain equality outside of a null set for each $t$; but by right continuity, we obtain indistinguishability. Thus, $H$ itself satisfies the theorem and by the monotone class theorem, the result holds for all bounded $H$.

Now suppose $H$ satisfies the hypotheses of the theorem. We let $f \in \mathscr{L}_{\infty}\left(E^{*}\right)$ and let $Y$ be a cadlag version of the martingale $E\left(f \mid \mathscr{F}_{t}\right)$. We denote the $L_{1}(E)$ valued measure $I_{V}$ by $m$. The real-valued measure $m_{f}$ defined on $\mathscr{O}$ is given by $m_{f}(B)=\langle f, m(B)\rangle=E[f \cdot m(B)]$. From $\left[1\right.$, p. 360], the $L_{1}\left(I_{V}\right)$ norm of $H$ is given by

$$
\|H\|_{L_{1}\left(I_{V}\right)}=\sup _{\|f\| \leq 1} \int|H| d\left|m_{f}\right|,
$$

where $\|f\|$ is the $L_{\infty}\left(E^{*}\right)$ norm of $f$. Thus, $H \in \mathscr{L}_{1}\left(I_{V}\right)$, and hence is stochastically integrable, if and only if this norm is finite. However, letting $A_{t}=\int^{t} 1_{B} d V_{s}$, for a fixed $B \in \mathcal{O}$, then

$$
\begin{aligned}
m_{f}(B) & =E\left[f\left(A_{\infty}-A_{\infty}^{p}+\int^{\infty}\left(1_{B}\right) d V_{s}^{p}\right)\right] \\
& =E\left[\int^{\infty} f d A_{s}\right]-E\left[\int^{\infty} f d A_{s}^{p}\right]+E\left[\int^{\infty} f^{p}\left(1_{B}\right) d V_{s}^{p}\right] \\
& =E\left[\int^{\infty}(f) d A_{s}\right]-E\left[\int^{\infty}(f) d A_{s}\right]+E\left[\int^{\infty}{ }^{p}(f)^{p}\left(1_{B}\right) d V_{s}^{p}\right] \\
& =E\left[\int^{\infty} \Delta Y_{s} d A_{s}\right]+E\left[\int^{\infty} Y_{s-}{ }^{p}\left(1_{B}\right) d V_{s}^{p}\right] \\
& =E\left[\int^{\infty} \Delta Y_{s} 1_{B} d V_{s}\right]+E\left[\int^{\infty}\left(Y_{s-} 1_{B}\right) d V_{s}^{p}\right] \\
& =E\left[\int^{\infty} \Delta Y_{s} 1_{B} d V_{s}\right]+E\left[\int^{\infty} Y_{s-} 1_{B} d V_{s}^{p}\right] .
\end{aligned}
$$

We see that $m_{f}$ can be written as the sum of two stochastic $P$-measures: $m_{f}(B)=\sigma(B)+\tau(B)$. Hence, $\left|m_{f}\right| \leq|\sigma|+|\tau|$. But since we take $\|f\| \leq 1$, 
$\|Y\| \leq 1$ also; hence, $|\sigma|(B) \leq E\left[\int^{\infty}\left\|\Delta Y_{s}\right\| 1_{B} d|V|_{s}\right] \leq E\left[\int^{\infty} 21_{B}\left|d V_{s}\right|\right]$ and $|\tau|(B) \leq E\left[\int^{\infty} 1_{B} d\left|V^{p}\right|_{s}\right]=E\left[\int^{\infty} 1_{B} d|V|_{s}^{p}\right]$, for all $f$.

Finally, we obtain

$$
\begin{aligned}
\|H\|_{L_{1}\left(I_{V}\right)} & \leq \int|H| d|\sigma|+\int|H| d|\tau| \\
& \leq 2 E\left[\int^{\infty}\left|H_{s}\right| d|V|_{s}\right]+E\left[\int^{\infty}\left|H_{s}\right| d|V|_{s}^{p}\right] \\
& =2 E\left[\int^{\infty}\left|H_{s}\right| d|V|_{s}\right]+E\left[\int^{\infty}{ }^{p}\left|H_{s}\right| d|V|_{s}\right] \\
& <\infty
\end{aligned}
$$

Lastly, if we let $H^{n}=H 1_{\{|H| \leq n\}}$, then $\left\{H^{n}\right\}$ are bounded, converge to $H$ in $L_{1}\left(I_{V}\right)$, and $\left\{H^{n} \cdot V\right\}$ satisfies the stated form of the theorem. By taking limits as in the monotone class argument, we see that $H \cdot V$ also satisfies the theorem.

\section{REFERENCES}

1. J. K. Brooks and N. Dinculeanu, Lebesgue-type spaces for vector integration, linear operators, weak completeness and weak compactness, J. Math. Anal. Appl. 54 (1976), 348-389.

2. C. Dellacherie and P. A. Meyer, Probabilities and potential, North-Holland, New York, 1982.

3. N. Dinculeanu, Vector measures, Pergamon Press, Oxford, 1967.

4. _ Vector-valued stochastic processes I. Vector measures and vector-valued stochastic processes with finite variation, J. Theoret. Probab. 1 (1988), 149-169.

5. —_ Vector-valued stochastic processes II. A Radon-Nikodym theorem for vector-valued processes with finite variation, Proc. Amer. Math. Soc. 102 (1988), 393-401.

6. __ Vector-valued stochastic processes III. Projections and dual projections, Seminar on Stochastic Processes, Birkhaüser, Boston, 1987.

7. —, Vector-valued stochastic processes. V. Optional and predictable variation of stochastic measures and stochastic processes, Proc. Amer. Math. Soc. 104 (1988), 625-631.

8. D. Neal, Characterization of O-summable processes, J. Theoret. Probab. 5 (1992), 585-596.

Department of Mathematics, Western Kentucky University, Bowling Green, KenTUCKY 42101

E-mail address: nealdk@wkuvx1.wku.edu 